\title{
ZERO-SUM PARTITION THEOREMS FOR GRAPHS
}

\author{
Y. CARO \\ Department of Mathematics \\ Haifa University \\ Oranim, Israel \\ I. KRASIKOV \\ Y. RODITTY \\ School of Mathematics \\ Tel-Aviv University \\ Ramat Aviv, Israel \\ Department of Mathematics \\ Beit-Berl College \\ Kfar-Saba, Israel
}

(Received November 13, 1992 and in revised form February 2, 1993)

ABSTRACT. Let $q=p^{n}$ be a power of an odd prime $p$. We show that the vertices of every graph $G$ can be partitioned into $t(q)$ classes $V(G)=\bigcup_{i=1}^{t(q)} V_{i}$ such that the number of edges in any induced subgraph $\left\langle V_{i}\right\rangle$ is divisible by $q$, where $t(q) \leq \frac{3}{2}(q-1)-\frac{(2(q-1)-1)^{\frac{1}{2}}}{4}+\frac{9}{8}$, and if $q=2^{n}$, then $t(q)=2 q-1$.

In particular, is is shown that $t(3)=3$ and $4 \leq t(5) \leq 5$.

KEY WORDS AND PHRASES. Zero-sum, partition, clique-number. 1991 AMS SUBJECT CLASSIFICATION CODES. 05C55.

\section{INTRODUCTION.}

Long ago, Gallai proved the following partition theorem:

THEOREM A. Let $G$ be a graph. Then there exists a partition $A \cup B=V(G), A \cap B=\phi$ such that in the subgraphs induced on $\langle A\rangle$ and $\langle B\rangle$ all the degrees are even (see [8] problem 5.17 or [6]).

Recently, this theorem received some attention ([4], [5]). A partial sample of the problems evolved from Gallai's theorem is:

PROBLEM 1: What is the maximum order of an induced subgraph $H$ of a graph $G$, with $n$ non-isolated vertices, such that in $\boldsymbol{H}$ all the degrees are even, respectively odd. In particular is it true that every tree on $n \geq 2$ vertices contains an induced subgraph on at least $\frac{2 n-2}{3}$ vertices in which all the degrees are odd?

For some results concerning this problem, see [4] and [5].

PROBLEM 2: Let $k \geq 2$ be an integer. Does there exist a constant $c=c(k)$, depending only on $k$, such that every graph can be partitioned into $c(k)$ vertex-disjoint classes $\bigcup_{i=1}^{c(k)} V_{i}=V(G)$ in such a way that in all the induced subgraphs $\left\langle V_{i}\right\rangle, 1 \leq i \leq c(k)$, all the degrees are divisible by $k$ ?

Clearly, Theorem A states that $c(2)=2$. The existence of $c(k)$ for $k \geq 3$ is an interesting open problem.

Motivated by Theorem A and the above problems, we consider the following related question:

PROBLEM 3: Let $k \geq 2$ be an integer. Does there exist a constant $t=t(k)$ such that every 
graph $G$ can be partitioned into $t(k)$ vertex-disjoint classes $\bigcup_{i=1}^{t(k)} V_{i}=V(G)$ in such a way that in each of the induced subgraphs $\left\langle V_{\imath}\right\rangle, 1 \leq i \leq t$ the number of edges is divisible by $k$ ?

Obviously an affirmative answer to Problem 2 (at least for $k$ odd) implies an affirmative answer to Problem 3 with $t(k) \leq c(k)$. Hence Problem 3 is weaker, and in fact more tractable, than Problem 2.

It is convenient for later purpose to introduce the following notations. Denote by $\phi(G, k)$ the smallest integer $m$ such that the hypergraph $G$ can be partitioned into $m$ classes $V(G)=\bigcup_{i=1}^{m} V_{i}$ in a way that $e\left(\left\langle V_{i}\right\rangle\right) \equiv 0(\operatorname{modk}), 1 \leq i \leq m$, where $e\left(\left\langle V_{i}\right\rangle\right)$ is the number of edges in the induced subhypergraph $V_{i}$. Observe that for every $k \geq 2$ and every hypergraph $G, \phi(G, k) \leq \chi(G)$, where $\chi(G)$ is the usual chromatic number of $G$, namely, the smallest number of colors such that each edge has vertices of at least two colors. Hence, with those notations Problem 3 is:

$$
\text { does sup } \phi(G, k)=t(k)<\infty \text { for a fixed } k \geq 2 \text { ? }
$$

Our main goal is to prove the existence of the constant $t(k)$ introduced in Problem 3. We shall do it explicitly for $k$ being a prime power, using the Baker-Schmidt Theorem [3], and indicate extensions to non-prime power moduli as well as to $r$-uniform hypergraphs. We shall then consider in detail some particular cases in which we were able to further improve upon our general upper bounds. In order to keep this introduction short and concise we shall only remark that our notations follow [2] and others will be presented when needed.

\section{PARTITION THEOREMS AND PROOFS.}

We start with an exact formulations of the main results.

THEOREM 1. Let $r, q \geq 2$ be positive integers.

(1) There exists a constant $t(q, r)$, depending only on $q$ and $r$, such that $\phi(H, q) \leq t(q, r)$, for every $r$-uniform hypergraph $\boldsymbol{H}$.

(2) If $r=2, q=2^{k}$ then $t(q, 2)=2 q-1$.

THEOREM 2. If $r=2, q=p^{n}, p$ is an odd prime, then

$$
t(q, 2) \leq \frac{3}{2}(q-1)-\frac{(2(q-1)-1)^{\frac{1}{2}}}{4}+\frac{9}{8}
$$

THEOREM 3. $t(3,2)=3$ and $4 \leq t(5,2) \leq 5$.

Before proving the theorems, we shall need the Baker-Schmidt theorem and some recent result of [1] which is given here in full detail in order to keep the paper self-contained.

THEOREM B (Baker-Schmidt [3]). Let $q$ be a prime power. If $s \geq d(q-1)+1$ and $h_{1}\left(x_{1}, \cdots, x_{s}\right), h_{2}\left(x_{1}, \cdots, x_{s}\right), \cdots, h_{1}\left(x_{1}, \cdots, x_{s}\right) \in Z\left[x_{1}, \cdots, x_{s}\right]$ are polynomials satisfying $h_{1}(0)=\cdots=h_{1}(0)=0$, and $\sum_{i=1}^{l} d e g h_{i} \leq d$, then there exists a binary vector $a \neq 0$ such that $h_{1}(a) \equiv \cdots \equiv h_{1}(a) \equiv 0(\bmod q)$.

Let $Z_{q}$ be the set of integers mod $q$ considered as an additive group.

THEOREM C (Alon and Caro [1]). Let $q$ be a prime power, and let $H=(V, E)$ by a hypergraph of rank $r$ (the size of the largest edge) and let $f: E \rightarrow Z_{q}$ be a $Z_{q}$-coloring of $E(H)$. Then there is a subset $U$ of $V$, where $|U| \geq|V|-r(q-1)$ such that $e\langle U\rangle \equiv 0(\bmod q)$. The same holds for $q$ a non-prime power provided $|U| \geq|V|-r q^{3} \log _{2} q$.

PROOF OF THEOREM C. For $q$ a prime power, let $m$ be the maximum integer such that there exists a set $M$ of $m$ vertices of $H$ so that $e\langle M\rangle \equiv 0(\operatorname{modq})$. If $m \geq|V|-r(q-1)$ there is nothing to prove. Otherwise, there are more than $r(q-1)$ vertices in $V \backslash M$. Define $W=V \backslash M$ and let $F$ be the set of all edges of $H$ that contain at least one vertex of $W$. Associate each vertex $w$ in $W$ with a variable $x_{w}$ and consider the following polynomial equation

$$
h=\sum_{e \in F} f(e) \prod_{w \in e} x_{w} \equiv 0(\bmod q) \text {. }
$$

The trivial vector $x_{w}=0$ for all $w \in W$, is clearly a solution of this equation. Since the degree of $h$ 
is at most $r$, Theorem $\mathrm{B}$ (for $l=1$ ) implies that there is a nontrivial solution in which each variable $x_{w}$ is either 0 or 1 . Let $W^{\prime}$ be the set of all the vertices $w$ for which $x_{w}=1$ in this solution. Note that for those vertices, the polynomial $h$ counts, $\bmod q$, the weight of the edges added to $e\langle M\rangle$, when adding $W^{\prime}$ to $M$. Thus, $e<M \cup W^{\prime}>\equiv 0(\bmod q)$, contradicting the maximality of $m$. This proves the assertion of the theorem for $q$ a prime power. For the second part of the theorem refer to [1]. $\square$

\section{PROOF OF THEOREM 1.}

(1) To prove this part, we adopt a greedy strategy, namely, we shall try to delete, in each stage, the largest possible induced subhypergraph whose number of edges is divisible by $q$. Using the above result, if $q$ is a prime power then $H$ contains an induced subhypergraph $G$, such that $|G| \geq|\boldsymbol{H}|-\boldsymbol{r}(q-1)$ and $|\langle\boldsymbol{H} \backslash G\rangle| \leq r(q-1)$. Thus, $\langle\boldsymbol{H} \backslash G\rangle$ can be decomposed into subsets of order at most $r-1$, which contain no edges. Since $H$ is $r$-uniform we conclude that in this case, $(q$ is a prime power $), t(q, r) \leq 1+\frac{r(q-1)}{r-1}=\frac{r q-1}{r-1}$. If $q$ is a non-prime power, then the same argument yields $t(q, r) \leq 1+\frac{r q^{3} \log _{2} q}{r-1}$.

(2) We already know by part (1) that $t(q, 2) \leq 2 q-1$ for $q=2^{k}$. It remains to present a graph that realizes the upper bound. Consider the complete graph $K_{2 q-1}$. Observe that for $1 \leq t \leq 2 q-1,\left(\begin{array}{c}t \\ 2\end{array}\right) \equiv 0(\bmod q), q=2^{k}$. Hence in a decomposition of $K_{2 q-1}$ each class consists a single vertex and we actually need $2 q-1$ classes. Hence $t\left(2^{k}, 2\right)=2^{k+1}-1$.

Before proving Theorem 2, we need a lemma.

A vertex-coloring of a graph $G$ is a function $g: V(G) \rightarrow\{1,2, \cdots, k\}$ in which adjacent vertices have different colors. A color-class is a class of vertices having the same color. By $\omega=\omega(G)$ we denote the clique-number of $G$.

LEMMA. Suppose $G$ is a graph on $n$ vertices. Let $\chi$ be a minimum vertex coloring of $G$ using $\chi(G)$ colors such that the number, $\left|X_{1}\right|$, of color classes of size one is as small as possible. Then,

(i) Each vertex in any color class $C,|C| \geq 3$ is adjacent to all vertices of $x_{1}$.

(ii) At least one vertex in any color class $C,|C|=2$ is adjacent to all vertices of $x_{1}$.

(iii) If $G \neq K_{n}$ then $\left|X_{1}\right| \leq \omega-1$.

(iv) If $G \neq K_{n}$ then $\phi(G, q) \leq \frac{n+\left|X_{1}\right|}{2} \leq \frac{n+\omega-1}{2}$.

PROOF.

(i) Suppose there were vertices $v \in C,|C| \geq 3$, and $w \in X_{1}$ such that $(v, w) \notin E(G)$. Then we can take $(v, w)$ as a color class, leaving $\chi(G)$ as before, but $\left|X_{1}\right|$ is reduced by one, a contradiction to the minimality of $\left|X_{1}\right|$.

(ii) Let $C=\{u, v\}$. If there is a vertex $w \in X_{1}$ such that $(w, u),(w, v) \notin E(G)$ then the vertices $\{w, u, v\}$ form a color class reducing $\chi(G)$.

Suppose now that there are vertices $w, z \in X_{1}$ such that $(w, u),(z, v) \notin E(G)$. Then we can take $\{w, u\}$ and $\{z, v\}$ as color classes, and again $\chi(G)$ is reduced. This contradiction shows that at least one of the vertices $u, v$ is adjacent to all $x_{1}$.

(iii) Clearly, $X_{1}$ forms a complete subgraph. If $G \neq K_{n}$ then by (i) or (ii), we can always add some vertex to $X_{1}$ to form a larger complete subgraph. Hence, $\left|X_{1}\right|+1 \leq \omega$. (iii).

(iv) As $\chi \leq\left|X_{1}\right|+\frac{n-\left|X_{1}\right|}{2}$ and for any $q \phi(G, q) \leq \chi(G)$ we obtain the required result using

In the proof of the Lemma we have used the trivial bound $\chi(G) \leq \frac{n+\omega-1}{2}$. Hence, we raise the following problem:

PROBLEM 4: What is the upper bound of $\chi(G)$ in the case when $\omega(G)=c n, c$ some constant? 
The relevant constant for our problens is about half.

PROOF OF THEOREM 2. Observe first that if $q$ is an odd integer, then $\left(\frac{q}{2}\right) \equiv 0(\bmod q)$ and $\left(\begin{array}{c}q+1 \\ 2\end{array}\right) \equiv 0(\bmod q)$. Consider a graph $G$ and estimate $\phi(G, q)$. Suppose we have deleted the largest subgraph assured by Theorem $C$. Then we are left with a collection $A$ of $2 q-l \leq 2 q-2$ vertices. We may assume w.l.o.g. that $|A|=2 q-2$ by adding isolated vertices. If $\langle A\rangle$ contains a complete graph $K_{t}, t \geq q$ then we may delete a $K_{q}$ from $\langle A\rangle$ and take all the remaining vertices as singles. We have at most $2+(2 q-2)-q=q$ classes which is smaller than the stated bound. Hence we may assume that the largest clique in $\langle A\rangle$ is of order at most $q-1$. Put $a=q-1$. Suppose that we have in $\langle A\rangle, k$ vertex disjoint independent sets of order 3. Delete those sets from $\langle A\rangle$, the resulting graph is denoted $A_{1}$. Consider a minimum vertex coloring of $A_{1}$ as in the Lemma. Call a vertex $v$ in $A_{1} \backslash X_{1}$ strong if it is adjacent to all vertices of $X_{1}$ and let $Y$ be the set of all strong vertices. Put $x=\left|X_{1}\right|$. Thus, $\omega\left(A_{1}\right) \geq x+\omega(\langle Y\rangle)$. By the lemma and the fact that $\left|A_{1}\right|=2 a-3 k$, we find:

(1) $\phi(G, q) \leq k+2 a-3 k+1=2(a-k)+1$.

Also, we have the trivial bound on $\phi(G, q)$,

(2) $\phi(G, q) \leq 1+k+\frac{2 a-3 k+x}{2}=1+a+\frac{x-k}{2}$.

Let $\beta_{0}(Y)$ be the cardinality of the maximal independent set in $\langle Y\rangle$. Then, $\beta_{0}(Y) \leq 2$ since independent sets of size 3 have been removed. We shall use a lower bound for $\beta_{0}(\bar{Y})$ obtained from Turan's Theorem (see [2]) and the fact that $\langle\bar{Y}\rangle$ is triangle free, namely,

(3) $\beta_{0}(\bar{Y}) \geq \max \left\{\frac{|Y|}{d+1}, d\right\} \geq|Y|^{\frac{1}{2}}-\frac{1}{2}$,

where $d$ is the average degree in $\langle Y\rangle$. Hence, $\omega(<Y>) \geq|Y|^{\frac{1}{2}}-\frac{1}{2}$. By (ii) of the lemma, $|Y| \geq \frac{2 a-3 k-x}{2}$. Hence,

(4) $x+\left(\frac{2 a-3 k-x}{2}\right)^{\frac{1}{2}}-\frac{1}{2} \leq x+\omega(Y) \leq a$.

Rewrite (4) as follows:

(5) $4 x^{2}-2 x(4 a+1)+4 a^{2}+1+6 k \geq 0$.

Then, either $8 a-24 k-3<0$, or

(6) $x \geq \frac{4 a+1+(8 a-24 k-3)^{\frac{1}{2}}}{4}$

or (7) $x \leq \frac{4 a+1-(8 a-24 k-3)^{\frac{1}{2}}}{4}$

Observe first that if $8 a-24 k-3<0$, that is $a<3 k+\frac{3}{8}$ then by (2) we obtain that $\phi(G, q) \leq \frac{4 a}{3}+\frac{5}{4}$ which is obviously better than the bound of the theorem. Otherwise, since $x \leq a(6)$, must be ignored. Now to obtain the required result substitute (7) with $k=0$ as the best choice, into (1). $\square$

REMARK 1.

(i) It is easy to show, by simple deletion of independent pairs, that $\phi(G, q) \leq \frac{3(q-1)}{2}$, for $q$ an odd prime. So the result of Theorem 2 is better than this trivial bound only for $q>11$.

(ii) The above bounds can be slightly improved using a result of Shearer [9] concerning the independent number of a triangle-free graph. The computations are simple but tedious.

PROOF OF THEOREM 3. Observe first that we may assume as before that after deleting the largest subgraph guaranteed by Theorem C, we are left with exactly $2 q-2$ vertices. So that for $q=3$ we have 4 vertices that remain. A direct checking of all graphs on 4 -vertices reveals that all of them are partitionable into at most two induced subgraphs in which the number of edges is divisible by 3 . Hence, $t(3,2) \leq 3$. 
The wheel $W_{5}$ :

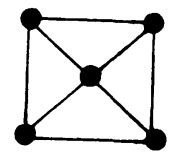

shows that indeed $t(3,2)=3$.

The case $q=5$ is more complicated since we have more than 12,000 graphs on 8 vertices and hand-checking is tedious. Our goal is to show that for every graph $G$ on 8 vertices $\phi(G, 5) \leq 4$. This would imply $t(5,2) \leq 5$. We split the proof into several cases:

(i) $\quad \omega \geq 5$.

In this case, we are done since $e\left(K_{5}\right)=10,\left|G \backslash K_{5}\right| \leq 3$ and $\phi(G, 5) \leq 4$.

(ii) $\boldsymbol{\omega} \leq 4$.

Then by Lemma $1,\left|x_{1}\right| \leq 3$.

(iia) If $\left|X_{1}\right|=0$ by (iv) of the Lemma we are done.

(iib) If $\left|X_{1}\right|=1$ then we have at most 7 vertices in at most 3 color classes with sizes at least 2 , so that again we are done.

(iic) If $\left|X_{1}\right|=2$ and we have a color class of size $\geq 3$, than take by Lemma, a $K_{4} \mid e$ (with 5 edges) and the rest of the (at most) four vertices contain at least one color class of size 2 and again we are done. Otherwise, all remaining color classes are of size 2. Then is is known from the Lemma 1 that there is at least one vertex in each color class that is adjacent to all vertices of $x_{1}$. Let $u$ and $v$ be such vertices of distinct color classes. If $(u, v) \notin E(G)$ then we have a $K_{4} \backslash e$ and we are done. Otherwise all such vertices are adjacent and together with $X_{1}$ create a complete subgraph of size 5 and we are in case (i).

(iid) If $\left|X_{1}\right|=3$, then either there is a single color class of size 5 and we are done or there is two color classes each of size at least two. Again, it is known from the Lemma that there is at least one vertex in each color class that is adjacent to all vertices of $x_{1}$. Let $u$ and $v$ be such vertices. If $(u, v) \notin E(G)$ then we have a $K_{4} \backslash e$ and we are done. Otherwise $\left\langle X_{1} \cup\{u, v\}>\right.$ induces a complete subgraph of size 5 and we are in case (i).

Now to show that $t(5,2) \geq 4$, consider the graph $K_{5 n+4}$. Take $V\left(K_{5 n+4}\right)=\bigcup_{i=1}^{4} A_{i}$, where, $\left|A_{1}\right|=5 n+1$ and $\left|A_{i}\right|=1, i=2,3,4$, and the number of edges of the induced subgraphs on the vertex sets $A_{i}$ is divisible by 5 . This gives $\phi\left(K_{5 n+4}, 5\right) \leq 4$. In order to show that $\phi\left(K_{5 n+4}, 5\right) \geq 4$, suppose that $V\left(K_{5 n+4}\right)=\bigcup_{i=1}^{3} A_{i}$, and the orders of the sets are $x_{1}, x_{2}, x_{3}$, respectively. Then,

(8) $x_{1}+x_{2}+x_{3}=5 n+4$.

On the other hand, each of the numbers $x_{1}, x_{2}, x_{3}$, should have been 0 or $1(\bmod 5)$. So that $(8)$ is impossible. $\square$

REMARK 2. We could not find a graph such that $\phi(G, 5)=5$. Its existence can give a complete answer in that case.

THEOREM 4. Let $q$ be an odd prime power and let $G$ be a graph such that all its induced subgraphs of order at most $2 q-2$ are perfect graphs. Then $\phi(G, q) \leq q$. In particular this holds for perfect graphs.

PROOF. By Theorem $C, G$ has a subgraph $H,|H| \geq|G|-2 q+2$ such that $e(H) \equiv 0(\bmod q)$. Clearly $|G \backslash H| \leq 2 q-2$. If $G \backslash H$ contains a clique $K_{t}, t \geq q$ take a $K_{q}$ and all the remaining vertices as singles to obtain a partition into at most $q$ classes. Otherwise by perfectness, $\phi(G \backslash H, q)=\chi(G \backslash H)=\omega(G \backslash H) \leq q-1$ which yields again a partition into at most $q$ classes.

We now characterize $\phi(G, 2)$ for every graph. 
THEOREM 5. Let $G$ be a graph. Then,

(i) $\phi(G, 2)=1$ iff $e(G) \equiv 0(\bmod 2)$.

(ii) $\phi(G, 2)=2$ iff $e(G) \equiv 1(\bmod 2)$ and there is a vertex of odd degree.

(iii) $\phi(G, 2)=3$ iff $e(G) \equiv 1(\bmod 2)$ and all degrees are even.

PROOF.

(i) Trivial.

(ii) Clearly, if $e(G) \equiv 1(\bmod 2)$ then $\phi(G, 2) \geq 2$ and if $\operatorname{deg} u \equiv 1(\bmod 2)$ then the required partition is $G \backslash\{u\},\{u\} \Rightarrow \phi(G, 2)=2$.

(iii) As $e(G) \equiv 1(\bmod 2)$ we have $\phi(G, 2) \geq 2$. We will show that $\phi(G, 2)=2$ is impossible and by $t(2,2)=3$ we must have $\phi(G, 2)=3$.

Indeed suppose $\phi(G, 2)=2$ and let $A, B$ be the vertex classes. As $e<A\rangle \equiv e\langle B\rangle \equiv 0(\bmod 2)$ and $e(G) \equiv 1(\bmod 2)$ we must have odd number of edges between $A$ and $B$. But this is possible iff an odd number of vertices of $A$ have an odd degree in $\langle A\rangle$ and for each of them there is odd number of edges connecting it to $\langle B\rangle$. But this is impossible since in $\langle A\rangle$ there will be an odd number of vertices of odd degree.

\section{REMARK 3.}

(1) By imitation of the proof of Theorem 2 we can show that if $q$ is prime power and $q \mid\left(\begin{array}{l}q \\ r\end{array}\right)$ then $t(q, r) \leq q+\frac{q-1}{r(r-1)}$.

(2) A deep theorem of Kierstead [7] states that if $G$ is both $K_{1,3}$-free and $K_{5} l e-$ free, then $\omega(G) \leq \chi(G) \leq \omega(G)+1$. Hence using the arguments of Theorem 4 we obtain that for such a graph $G$ and for $q$ an odd prime power $\phi(G, q) \leq q+1$.

Finally, we pose the following conjecture.

CONJECTURE. If $q \neq 2^{k}$ then $t(q, 2) \leq q(1+o(1))$.

ACKNOWLEDGEMENT. We would like to thank the referee for his careful reading and helpful remarks.

\section{REFERENCES}

1. ALON, N. \& CARO, Y., On three zero-sum Ramsey-type problems, J. of Graph Theory, in press.

2. BOLLOBAS, B., Extremal Graph Theory, Academic Press, New York, 1978.

3. BAKER, R.C. \& SCHMIDT, W.M., Diophantine problems in variables restricted to the values 0 and 1, J. Number Theory 12 (1980), 460-486.

4. CARO, Y., On induced subgraphs with odd degrees, Discrete Mathematics, to appear.

5. CARO, Y.; KRASIKOV, I. \& RODITTY, Y., On induced subgraphs of trees, with restricted degrees, Discrete Mathematics, to appear.

6. CHEN, W.K., On vector spaces associated with graphs, SIAM J. Appl. Math. 20 (1971), 526-529.

7. KIERSTEAD, H.A., On the chromatic index of multigraphs without large triangles, $J$. Comb. Theory (Ser B) 36 (1984), 156-160.

8. LOVASZ, L., Combinatorial Problems and Exercises, North-Holland, Amsterdam, 1979.

9. SHEARER, J.B., A note on the independence number of triangle-free graphs II, J. of Comb. Theory (Ser B) 53 (1991), 300-307. 


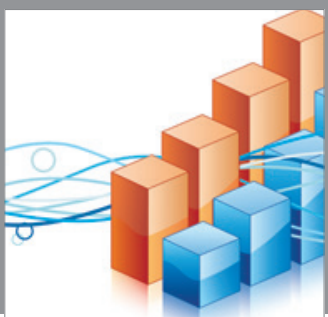

Advances in

Operations Research

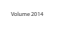

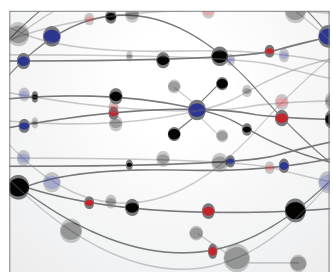

\section{The Scientific} World Journal
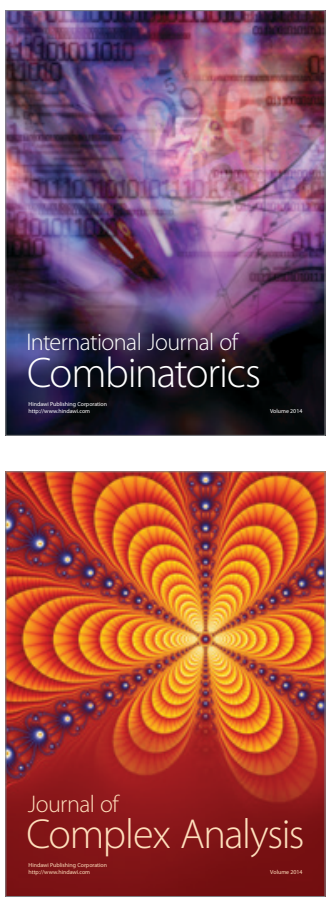

International Journal of

Mathematics and

Mathematical

Sciences
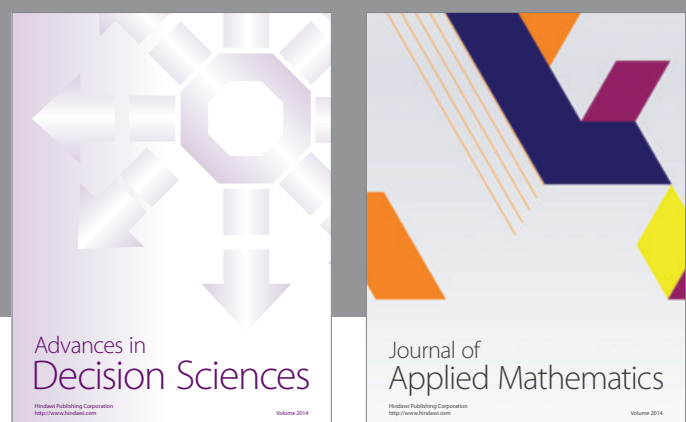

Journal of

Applied Mathematics
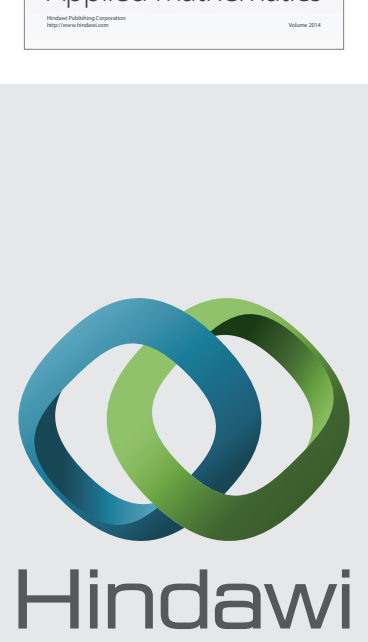

Submit your manuscripts at http://www.hindawi.com
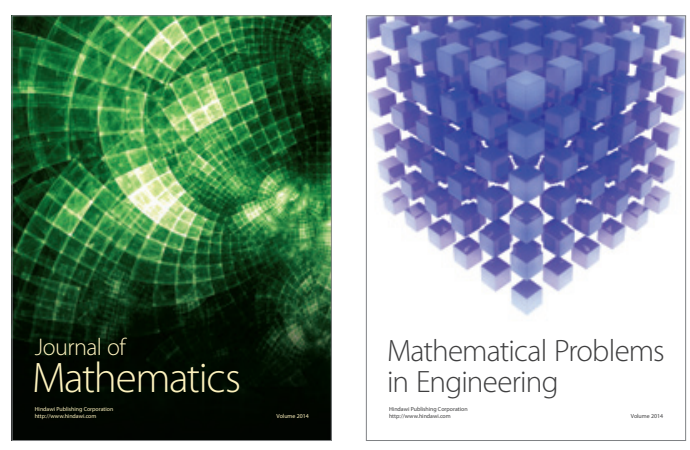

Mathematical Problems in Engineering
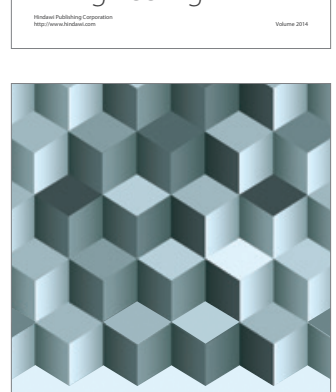

Journal of

Function Spaces
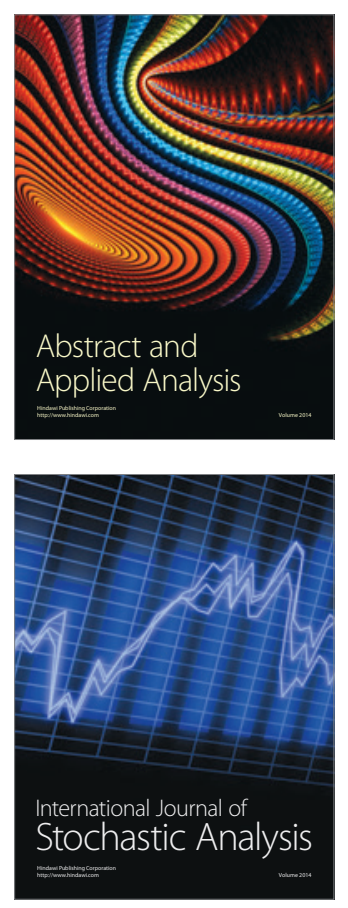

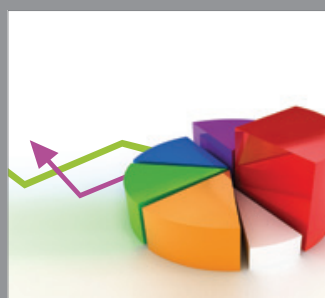

ournal of

Probability and Statistics

Promensencen
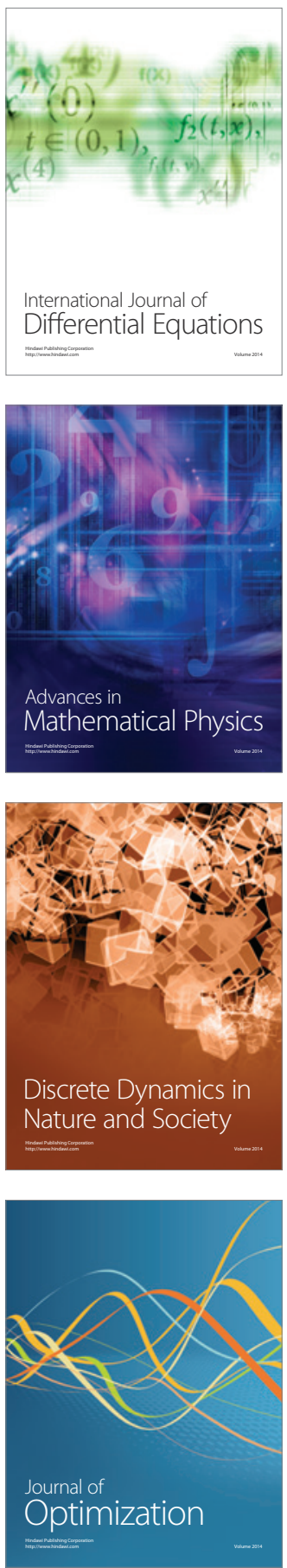